\title{
Incidence Rate, Risk Factors and Outcome of Premature Rupture of Membranes (PROM) at Zagazig University Hospitals
}

\author{
Ali El-Shabrawy Ali, Wael Sabry Nossair, Randa Mohamed Reda Abdel Maaboud, Safaa Abdelsalam Ibrahem \\ Department, Obstetrics and Gynecology, Faculty of Medicine, Zagazig University, Egypt. \\ Corresponding Author: Randa Mohamed Reda Abdel Maaboud, Mobile: (+20) 01126749855,
}

Email: randamohamed007@ gmail.com

\begin{abstract}
Background: Premature rupture of membranes (PROM) is a common pregnancy complication and is associated with significant risks of fetal and neonatal morbidity and mortality.

Objective: This study aimed to measure the incidence rate of preterm premature rupture of membranes (PPROM) among pregnant women attending Zagazig University Hospital, and to identify the risk factors associated with PROM and fetal/neonatal outcomes.

Methods: This cross-sectional study was conducted at Zagazig University Hospitals. It included 69 women with PROM. The study was conducted from July 2019 to January 2020. All patients were subjected to detailed history taking, general examination and laboratory investigations.

Results: There were 46 mother gave birth of healthy babies with good Apgar score (66.7\%), 15 with babies needed $\mathrm{O}_{2}$ incubators (21.7\%) and 8 with babies that were put on ventilators (11.6\%). There were 68 mothers with no bad outcomes and 1 with chorioamnionitis. The risk factors of the cases showed that there were 15 with no risk factor $(21.7 \%), 8$ with previous PROM (11.6\%), 6 with multi pregnancy (8.7\%), 12 with antepartum (17.4\%), 16 with infections (23.2\%) and 12 with chronic diseases $(17.4 \%)$.

Conclusions: we concluded that younger, illiterate parturient women were found to be provoking factors to increased PPROM. Such hazards may affect both maternal and neonatal outcome such as infection, maternal distress, fetal distress, increase operative delivery, as well as need for neonatal intensive care unit care in more than $50 \%$ of the neonates.
\end{abstract}

Key words: Premature rupture of membranes, Abortion, Risk Factors.

\section{INTRODUCTION}

Premature rupture of the membranes (PROM) is usually defined as rupture of membranes at any time before the onset of uterine contractions. PROM which occurs prior to 37 weeks of gestation is referred as preterm premature rupture of membranes (PPROM), whereas PROM, which occurs after 37 weeks of gestation, is referred as term premature rupture of membranes. The latent period is defined as the duration from rupture of the membranes until the onset of true labor $^{(\mathbf{1})}$.

The prevalence of PROM varies in different countries and populations, and many factors affect its occurrence. Therefore, its etiology is complex and multifactorial. Two-thirds of PROM cases occur spontaneously or for unknown reasons. However, other cases are due to structural defects in the membrane due to deficiency of collagen content in the membrane, protrusion of the membrane due to isthmus-cervical incompetence and activation of catabolic enzymes such as collagenase. The fetal membranes weaken due to enzymatic degeneration in inflammatory or infectious processes, mechanical stresses, and secretion of proteolytic enzymes from cervicovaginal flora or infection of amniotic fluids ${ }^{(2)}$. One of the main causes of the PROM occurrence is infection (often bacterial infection) that stimulates the release of proinflammatory cytokines from decidua and amniotic membranes. Therefore, many bioactive materials, such as prostaglandins and metalloproteases are released. Prostaglandins stimulate uterine contractions, and metalloproteases cause cervical ripening, and ultimately cause the rupture of membrane ${ }^{(\mathbf{1})}$.

The risk factors for PROM include maternal ones, including the history of PROM in previous pregnancies (the risk of recurrence of $16 \%$ to $32 \%$ in comparison with the risk of $4 \%$ in non-complicated term pregnancies), vaginal bleeding before delivery, longterm use of steroids, vascular collagen disorders such as EhlersDanlos syndrome, systemic lupus erythematosus, abdominal trauma, preterm labor history, cigarette smoking, drug abuse, anemia, low BMI (under 19.8 $\mathrm{kg} / \mathrm{m}^{2}$ ), food deficiencies including ascorbic acid and copper, low socioeconomic status, history of hypertension, abortion, cesarean section, black race/ethnicity, access to hospital care services, marital status, parity, history of preterm labor and exposure to diethylstilbestrol in the uterus. Other risk factors in this group are related to pregnancy complications such as gestational diabetes or overt diabetes, maternal weight gain, invasive procedures such as cerclage and amniocentesis ${ }^{(3)}$.

PPROM is associated with a prenatal morbidity and mortality rate in more than $20 \%$, and the outcomes are primarily dependent on the gestational age at delivery. The key for reducing the adverse effects of PPROM is to make a prompt diagnosis, admission and start antibiotic coverage ${ }^{(4)}$. The aim of the present study 
was to measure the incidence rate of PPROM among pregnant women attending Zagazig University Hospital, and to identify the risk factors associated with PROM and fetal/neonatal outcomes.

\section{PATIANT AND METHODS}

This cross-sectional study was carried out at ER Department, Zagazig University Hospitals for vaginal deliveries, from July 2019 till January 2020, on 69 women with premature rapture of membrane.

Sample size: The total women attending Zagazig University from July 2019 till January 2020 hospital was 8400 and prevalence rate of premature rapture of membrane was $4.7 \%$ so sample is 69 women. Sample was calculated using open EPI program with confidence interval $95 \%$ and the power of the test was $80 \%$.

Inclusion criteria: Patients presented by ROM. Gestational age (27-37wks). Patients presented by chorioamnionitis (fetal tachycardia-maternal fever). Clear amniotic fluid. No threatened PTL. Patient present with chronic diseases (DM \& HTN).

Exclusion criteria: Rapture of membrane after 37 wks. Congenital fetal anomalies. Placental abnormalities. Presence of fetal stress sign. Meconium liquor stain. Antepartum hemorrhage.

\section{Ethical approval:}

Written informed consent was obtained from all patients. The study was approved by the Research Ethical Committee, Faculty of Medicine, Zagazig University. The work was carried out for studies involving humans in accordance with the World Medical Association's Code of Ethics (Helsinki Declaration).

\section{Method:}

All patients were subjected to detailed history taking including passage of liquor from vagina, gravidity, parity, abortion [duration of pregnancy, mode of termination (spontaneous, induced)], history of PROM, BMI, weight gain during pregnancy, mode of delivery, last delivery, delivery complications, newborn (male-female, died-alive), menstrual history and general examination.

The examination of sterile speculum was done and the presence of amniotic fluid, which was then collected on slide and examined under microscope. Amniotic fluid culture and urine culture were done. All cases were administered with prophylactic IV antibiotics. In all the cases, a 4th hourly record of pulse of mothers, blood pressure and temperature was maintained and delivery was conducted within 24 hours. Fetal distress cases were delivered by emergency caesarean section. Patients were followed till their delivery and postnatal data regarding mode of delivery, fetal weight, APGAR score and neonatal outcomes were recorded on the perform.

Main outcome measures were prevalence of PPROM before 37 weeks. Its association with maternal demographic and obstetrical variables along with mode of delivery, low birth weight perinatal morbidity and mortality and maternal morbidity.

Laboratory investigations included CBC, CRP, Nitrazine test: colour turn to deep blue from yellow due to alkalinity of amniotic fluid, Fern test: visualization of fern-like pattern on glass due to presence of protein and $\mathrm{NaCl}$ crystals. US to detect age, weight, presentation, viability, index. Amniotic fluid culture \& urine culture were done.

Maternal outcomes: Mode of delivery (spontaneous vaginal or CS delivery), presence of clinical chorioamnionitis, which is characterized by maternal fever $\left(>39^{\circ} \mathrm{c}\right)$ accompanied by at least two of the following signs: maternal or fetal tachycardia, maternal leukocytosis, uterine tenderness, or foulsmelling amniotic fluid.

Fetal/Neonatal outcome: Birth weight (in $\mathrm{Kg}$ ), alive and well, alive but needed neonatal intensive care unit (NICU) admission, neonatal death or others (eg. neonatal infection or jaundice).

\section{Statistical methods}

Analysis of data was done using Statistical Program for Social Science version 20 (SPSS Inc., Chicago, IL, USA). Quantitative variables were described in the form of mean and standard deviation. Qualitative variables were described as number and percent. Student t test was performed. Qualitative variables were compared using chi-square $\left(\mathrm{X}^{2}\right)$ test or Fisher's exact test when frequencies were below five. Pearson correlation coefficients were used to assess the association between two normally distributed variables. When a variable was not normally distributed, $\mathrm{P}$ value $\leq 0.05$ is considered significant.

\section{RESULTS}

Table (1) showed that the studied cases $(n=69)$ had mean age of $26.55 \pm 6.15$ with range of $17-43$ and median 26 (24-31) years. The gravidity of the cases $(n=69)$ had mean of $2.78 \pm 1.82$ with range of $1-8$ and parity mean of $1.3 \pm 1.33$ with range of $0-6$. There were 49 cases (previously pregnant) had abortion between 0 6 times with average $0.69 \pm 0.16$. BMI of the cases had mean of 28.5 with range of $24-30 \mathrm{~kg} / \mathrm{m}^{2}$. The gestational age at PROM (wks.) of studied cases was $34.57 \pm 2.7$ with range of 28-39.71 weeks. Latent period (hours) was $9 \pm 6.16$ with range of $2-42$ and the gestational age at termination (wks.) was $35.85 \pm 2.57$ with range of 29.14-40.29. 
Table (1): Descriptive analysis of the studied cases regarding basic characteristic data of the studied groups $(n=69)$

\begin{tabular}{|c|c|c|c|c|}
\hline & No. & Min. - Max. & Mean \pm SD. & Median (IQR) \\
\hline \hline Age (years) & 69 & $17.0-43.0$ & $26.55 \pm 6.15$ & $26.0(24.0-31.0)$ \\
\hline BMI & 69 & $24-30$ & 28.52 .1 & \\
\hline Gravidity & 69 & $1.0-8.0$ & $2.78 \pm 1.82$ & $2.0(2.0-4.50)$ \\
\hline Parity & 69 & $0.0-6.0$ & $1.30 \pm 1.33$ & $1.0(1.0-3.0)$ \\
\hline Abortion & 49 & $0.0-6.0$ & $0.69 \pm 0.16$ & $0.0(0.0-1.0)$ \\
\hline Gestational age at PROM (wks.) & 69 & $28.0-39.71$ & $34.57 \pm 2.70$ & $35.0(32.36-36.57)$ \\
\hline Gestational age at termination (wks.) & 69 & $29.14-40.29$ & $35.85 \pm 2.57$ & $36.64(33.79-37.57)$ \\
\hline
\end{tabular}

Table (2) showed that the risk factors of the cases were: 15 with no risk factor (21.7\%), 8 with previous PROM $(11.6 \%), 6$ with multi pregnancy (8.7\%), 12 with antepartum (17.4\%), 16 with infections $(23.2 \%)$ and 12 with chronic diseases $(17.4 \%)$.

Table (2): Distribution of the studied cases regarding risk factors $(n=69)$

\begin{tabular}{|c|c|c|}
\hline \multicolumn{1}{|c|}{ Risk factor } & No. & \% \\
\hline None & 15 & 21.7 \\
\hline Previous PROM & 8 & 11.6 \\
\hline Multiple pregnancy & 6 & 8.7 \\
\hline Antepartum He & 12 & \\
-Placenta previa & 9 & 17.4 \\
-Placenta accrete & 3 & \\
-Accidental Hge & 0 & 23.2 \\
-Vasa previa & 0 & \\
\hline Chronic disease & 16 & 17.4 \\
DM & 12 & \\
HTN & 6 & \\
Liver \& kidney disorders & 6 & \\
\hline
\end{tabular}

Table (3) showed that there were 63 cases had single baby (91.3\%), 5 cases had twins (7.2\%) and 1 case had triplets $(1.4 \%)$. There were 43 male babies $(56.6 \%)$ and 33 female babies $(43.4 \%)$.

Table (3): Distribution of the studied cases according to number of feti during pregnancy

\begin{tabular}{|c|c|c|}
\hline & No. & \% \\
\hline \hline Number of fetus & & \\
\hline Single & 63 & 91.3 \\
\hline Twins & 5 & 7.2 \\
\hline Triplets & 1 & 1.4 \\
\hline Total of cases & \multicolumn{2}{|c|}{69} \\
\hline
\end{tabular}

Table (4) showed that among the studied cases, there were 52 cases had cesarean delivery (75.4\%) and 17 cases had normal vaginal delivery $(24.6 \%)$.

Table (4): Distribution of the studied cases according to mode of delivery $(n=69)$

\begin{tabular}{|c|c|c|}
\hline Procedure & No. & \% \\
\hline \hline CS & $\mathbf{5 2}$ & \\
Abnormal position & $\mathbf{5}$ & \\
Fetal distress & $\mathbf{1 6}$ & $\mathbf{7 5 . 4}$ \\
Birth defect & $\mathbf{2}$ & \\
Repeat section & $\mathbf{2 0}$ & \\
Chronic health condition & $\mathbf{1}$ & \\
Cord prolapse & $\mathbf{3}$ & 24.6 \\
Cephalo pelvic disproportion & $\mathbf{5}$ & \\
\hline NVD & 17 & \\
\hline
\end{tabular}


Table (5) showed that among the studied cases, there were 46 mothers gave birth of healthy babies with good Apgar score (66.7\%), 15 with babies needed $\mathrm{O}_{2}$ incubators (21.7\%) and 8 with babies that were put on ventilators (11.6\%).

Table (5): Distribution of the studied cases according to neonatal outcomes

\begin{tabular}{|c|c|c|c|}
\hline & score & No. & \% \\
\hline \hline Good Apgar score & $(7-10)$ & & 66.7 \\
At 1 $\mathbf{s t}^{\text {minutes }}$ & $7-8$ & 46 & \\
At 5 $5^{\text {st }}$ minutes & $8-10$ & & \\
\hline NICU & & & 21.7 \\
\hline O $^{2}$ mask & $<7$ & 15 & 11.6 \\
\hline On ventilator & $<4$ & 8 & \\
\hline
\end{tabular}

Table (6) showed that the outcomes of mothers were: 52 (75.4\%) with C.S, 17(24.6\%) with NVD, 66 mothers with no bad outcomes and 1 with chorioamnionitis and $2(2.8 \%)$ with accidental hg.

Table (6): Distribution of the studied cases according to maternal outcomes

\begin{tabular}{|l|c|c|}
\hline & No. & \% \\
\hline \hline C.S & 52 & 75.4 \\
\hline Chorioamnionitis & 1 & 1.4 \\
\hline Abruption placenta(accidental hg) & 2 & 2.8 \\
\hline NAD (No abnormalities detected) & 66 & 95.6 \\
\hline
\end{tabular}

Table (7) showed that among cases with no risk factors, 11 gave birth of healthy feti with good Apgar, 2 feti needed $\mathrm{O}_{2}$ incubator and 2 feti were put on ventilator. Among cases with previous PROM, 4 gave birth of healthy babies with good Apgar, 1 with fetus needed $\mathrm{O}_{2}$ incubator and 3 with feti were put on ventilator. Among cases with multiple pregnancies, 0 gave birth of healthy babies with good Apgar, 4 feti needed $\mathrm{O}_{2}$ incubator and 2 feti were put on ventilator. Among cases with antepartum risk factors 10 gave birth of healthy feti with good Apgar, 2 fetuses needed $\mathrm{O}_{2}$ incubator and no fetus was put on ventilator. Among cases with infections, 13 gave birth of healthy fetuses with good Apgar, 2 fetuses needed $\mathrm{O}_{2}$ incubator and 1 fetus was put on ventilator. Among cases with chronic diseases, 8 gave birth of healthy fetuses with good Apgar, 4 fetuses needed $\mathrm{O}_{2}$ incubator and no fetus was put on ventilator. This table showed that there was statistically significant difference between outcomes of fetuses regarding risk factors of the mothers.

Table (7): Relation between outcome and risk factor $(\mathrm{n}=69)$

\begin{tabular}{|c|c|c|c|c|c|c|c|c|}
\hline \multirow{3}{*}{ Risk factor } & \multicolumn{6}{|c|}{ Outcome } & \multirow{3}{*}{$\mathbf{x} 2$} & \multirow{3}{*}{${ }^{\mathrm{MC}} \mathbf{p}$} \\
\hline & \multicolumn{2}{|c|}{$\begin{array}{c}\text { Good Apgar score } \\
\qquad(n=46)\end{array}$} & \multicolumn{2}{|c|}{$\begin{array}{c}\mathrm{O}_{2} \text { Incubator } \\
(\mathrm{n}=15)\end{array}$} & \multicolumn{2}{|c|}{$\begin{array}{l}\text { On ventilator } \\
(n=8)\end{array}$} & & \\
\hline & No. & $\%$ & No. & $\%$ & No. & $\%$ & & \\
\hline None & 11 & 23.9 & 2 & 13.3 & 2 & 25.0 & & \\
\hline Previous PROM & 4 & 8.7 & 1 & 6.7 & 3 & 37.5 & & \\
\hline Multiple pregnancy & 0 & 0.0 & 4 & 26.7 & 2 & 25.0 & & \\
\hline $\begin{array}{l}\text { Antepartum } \\
\text { Placenta previa } \\
\text { Placenta accrete } \\
\text { Accidental He } \\
\text { Vasa previa }\end{array}$ & 10 & 21.7 & 2 & 13.3 & 0 & 0.0 & $20.997^{*}$ & $0.004^{*}$ \\
\hline \begin{tabular}{|l|} 
Infections \\
\end{tabular} & 13 & 28.3 & 2 & 13.3 & 1 & 12.5 & & \\
\hline $\begin{array}{l}\text { Chronic disease } \\
\text { DM } \\
\text { HTN } \\
\text { Liver \& kidney disorders }\end{array}$ & 8 & 17.4 & 4 & 26.7 & 0 & 0.0 & & \\
\hline
\end{tabular}

$\chi^{2}$ : Chi square test MC: Monte Carlo

Statistically significant at $\mathrm{p} \leq 0.05$

p: $p$ value for association between different categories

Table (8) showed that for cases with babies of good Apgar, their latent period was $5.89 \pm 1.64$ with range of 3.07.0 and median of $5 \mathrm{hrs}$. For cases with babies that needed $\mathrm{O}_{2}$ incubator, there latent period was $7.73 \pm 2.39$ with range of 3.0-12.0 and median of $7 \mathrm{hrs}$. For cases with babies that were put on ventilator, their latent period was $10.25 \pm 3.37$ with range of 2.0-15.0 and median of 10 hrs. There was high statistically significant difference between the outcomes of babies concerning the latent period. 
Table (8): Relation between outcome and latent period (hours) $(n=69)$

\begin{tabular}{|c|c|c|c|c|c|}
\hline \multirow{2}{*}{$\begin{array}{l}\text { Latent period } \\
\quad \text { (hours) }\end{array}$} & \multicolumn{3}{|c|}{ Outcome } & \multirow[b]{2}{*}{$\mathbf{H}$} & \multirow[b]{2}{*}{$\mathbf{p}$} \\
\hline & $\begin{array}{c}\text { Good Apgar score } \\
(n=46)\end{array}$ & $\begin{array}{c}\mathrm{O}_{2} \text { Incubator } \\
(\mathrm{n}=15)\end{array}$ & $\begin{array}{c}\text { On ventilator } \\
(\mathbf{n}=8)\end{array}$ & & \\
\hline Min. - Max. & $3.0-7.0$ & $3.0-12.0$ & $2.0-15.0$ & \multirow{3}{*}{17.21} & \multirow{3}{*}{$<0.001 * *$} \\
\hline Mean \pm SD & $5.89 \pm 1.64$ & $7.73 \pm 2.39$ & $10.25 \pm 3.37$ & & \\
\hline Median & 5.0 & 7.0 & 10.0 & & \\
\hline
\end{tabular}

$\mathrm{H}$ : H for Kruskal Wallis test

p: $\mathrm{p}$ value for association between different categories. *: Statistically significant at $\mathrm{p} \leq 0.05$

\section{DISCUSSION}

The studied cases $(n=69)$ had mean age of $26.55 \pm$ 6.15 with range of 17-43 and median of 26 (24-31) years, there were 34 cases had age less than or equal to 25 years $(49.3 \%)$ and 35 cases had age more than 25 years $(50.7 \%)$. Our results are in agreement with study of Negara et al. ${ }^{(5)}$ as they reported that the mean age of the cases group was $26.59 \pm 6.49$ years. In the study of Brian and Mercer ${ }^{(6)}$, they found that the incidence of PROM was most common in the 21-30-years age group.

The present study showed that the gravidity of the cases $(n=69)$ had mean of $2.78 \pm 1.82$ with range of $1-8$ and parity mean of $1.3 \pm 1.33$ with range of $0-6$. There were 49 cases (previously pregnant) had abortion between $0-6$ times with average $0.69 \pm 1.09$. The gestational age at PROM (wks.) of studied cases $(n=69)$ was $34.57 \pm 2.7$ with range of 28-39.71. Latent period (hours) was $9 \pm 6.16$ with range of $2-42$ and the gestational age at termination (wks.) was $35.85 \pm 2.57$ with range of 29.14-40.29. Among the studied cases, 63 cases had single baby $(91.3 \%), 5$ cases had twins $(7.2 \%)$ and 1 case had triplets $(1.4 \%)$. There were 43 male babies (56.6\%) and 33 female babies (43.4\%). Based on gravida, study by Brian and Mercer ${ }^{(6)}$ found that the highest incidence of PROM occurred in primigravida group that was 87 cases (41.05\%). Equal with what reported by Okeke $\boldsymbol{e t}$ al. ${ }^{(7)}$ on a retrospective study in Nigeria that the highest incidence of preterm PROM cases occurred in primigravida group (29.1\%). Patil $\boldsymbol{e t}$ al. ${ }^{(8)}$ in his study reported 53\%. Noor et al. ${ }^{(9)}$ reported that the incidence of PROM was also highest in primigravida cases $(42.2 \%)$. Other studies have reported that the incidence of PROM also occurred in primigravida of $68.2 \%, 52 \%, 69.7 \%{ }^{(10)}$.

The current study showed that there were 52 cases had cesarean delivery (75.4\%) and 17 cases had normal vaginal delivery (24.6\%). Our results are supported by study of Sae-Lin and Wanitpongpan (11) as they reported that $39.7 \%$ of their cases had cesarean section. Furthermore, Noor et $\boldsymbol{a l} .{ }^{(9)}$ found that normal vaginal delivery was the commonest mode of delivery ( 56 cases, $65.86 \%$ ), while instrumental delivery rate was $20 \%$ (17 cases) and caesarean section rate was $14 \%$ (12 cases).

In the study in our hands, there were 46 mothers gave birth of healthy babies with good Apgar score (66.7\%), 15 with babies needed $\mathrm{O}_{2}$ incubators (21.7\%) and 8 with babies that were put on ventilators (11.6\%). Concerning the outcomes of mothers, there were 68 mothers with no bad outcomes and 1 with chorioamnionitis. Our results are supported by study of Bouvier et al $^{(\mathbf{1 2})}$ as they reported that the complications associated with PPROM were oligohydramnios (aOR: 4.17 (2.37-7.35)), abruptio placentae (aOR: 4.28 (1.879.78)), cesarean (aOR: 1.41 (1.02-1.96)), Apgar $5^{\prime}<4$ (aOR: 23.32 (7.04-77.19)), birth weight $<2500 \mathrm{~g}$ (aOR: 47.74 (32.52-70.08)), stillbirth (1.1\% in PPROM group versus $0 \%$ in control group, $\mathrm{p}<0.0001)$, neonatal jaundice (aOR: 3.25 (2.20-4.80)), hospitalization of mother (aOR: 1.75 (1.15-2.65)), and admission at the neonatal intensive care unit (aOR: 17.12 (12.2323.98)). All these complications were also associated with SPL with IM (spontaneous preterm labor with intact membranes).

The present study showed that the risk factors of the cases were: 15 with no risk factor $(21.7 \%), 8$ with previous PROM (11.6\%), 6 with multi-pregnancy $(8.7 \%), 12$ with antepartum (17.4\%), 16 with infections (23.2\%) and 12 with chronic diseases (17.4\%). Among cases with no risk factors, 11 gave healthy babies with good Apgar, 2 with babies in need for $\mathrm{O}_{2}$ incubator and 2 with babies that were put on ventilator. Among cases with previous PROM, 4 gave healthy babies with good Apgar, 1 with babies needed $\mathrm{O}_{2}$ incubator and 3 with babies that were put on ventilator. Among cases with multiple pregnancy, 0 gave birth of healthy babies with good Apgar, 4 with babies needed $\mathrm{O}_{2}$ incubator and 2 with babies that were put on ventilator. Among cases with antepartum, 10 gave birth of healthy babies with good Apgar, 2 with babies needed $\mathrm{O}_{2}$ incubator and 0 with babies that were put on ventilator. Among cases with infections, 13 gave healthy babies with good Apgar, 2 with babies needed $\mathrm{O}_{2}$ incubator and 1 with babies that were put on ventilator. Among cases with chronic disease, 8 gave birth of healthy babies with good Apgar, 4 with babies needed $\mathrm{O}_{2}$ incubator and 0 with babies that were put on ventilator. There was statistically significant difference between outcomes of the babies as regard risk factors of the mothers.

Several studies from USA, Sweden, India, Thailand, Egypt, Nigeria and Uganda revealed that previous PROM was a significant risk factor for premature rupture of membranes ${ }^{(3)}$. This study also 
showed that previous PROM was the strongest risk factor for premature ruptures of membranes. Women who had previous PROM were 4.45 more likely to develop PROM with adjusted odds ratio (AOR) 4.45 (CI: 1.87, 10.6). This might be due to untreated genitourinary infection and a short cervical length. In addition, obstetric problems are recurrent by nature.

In our study vaginal bleeding, accident, gestational age, gravidity, parity, presentation, polyhydramnious, multiple pregnancy, anemia and pregnancy-induced hypertension were not found to be a significant predictor of premature rupture of membranes. This is discordant with findings from researches conducted in Sweden, Lithuania and India ${ }^{(3)}$. Bouvier et al. ${ }^{(12)}$ confirmed the most known risk factors for PPROM were BMI $<18.5 \mathrm{~kg} / \mathrm{m}^{2}$, history of PPROM or prematurity, nulliparity, multiple pregnancies, low level of education, and infections. These results, as well as the percentage of PPROM (2.7\%), validated the cohort, which is comparable with others ${ }^{(\mathbf{1 3})}$. Infections, history of prematurity, and multiple pregnancies are known risk factors of both PPROM and also sPL with IM. The association of infection with prematurity at large is in line with recent hypotheses presenting infection in PPROM as a downstream event rather than a causal factor ${ }^{(14)}$. Recent reports indicated that PPROM might be associated with the presence of sterile inflammation in the fetal membranes. Sterile inflammation may be responsible for the link between GDM and PPROM ${ }^{(15)}$. Although infection has been accepted as a major risk factor of PPROM, many of previous preventive strategies by antibiotics seemed unsatisfactory and did not help to reduce the incidence ${ }^{(\mathbf{1 6})}$.

Complications of PROM can affect either the infants or maternal outcomes. Maternal complications such as chorioamnionitis are found in 9\% of pregnancies with premature rupture of membranes. In preterm pregnancies, the incidence was greater between $13-60 \%{ }^{(17)}$.

The current study showed that for cases with babies of good Apgar, their latent period was $8.89 \pm 6.64$ with range of 3-42 and median of $7 \mathrm{hrs}$. For cases with babies needed $\mathrm{O}_{2}$ incubator, their latent period was $9.73 \pm 5.69$ with range of 3-22 and median of $7 \mathrm{hrs}$. For cases with babies that were put on ventilator, their latent period was $8.25 \pm 4.37$ with range of 3-15 and median $7 \mathrm{hrs}$. There was no statistically significant difference between the outcomes of babies as regards the latent period. Tchirikov et al. ${ }^{(18)}$ found that the lack of information on the prolongation of pregnancy after PPROM (latency period) could be considered as a potential limitation. Indeed, a latency period could be associated with a higher incidence of complications. The complications associated with PPROM, such as oligohydramnios, abruption placentae, Apgar $5^{\prime}<4$, weight $<2500$ g, stillbirth, neonatal jaundice, and hospitalization of neonates in NICU, are not related to PPROM per se but are associated with prematurity ${ }^{\left({ }^{(19)}\right.}$.
PROM increased the incidence of RDS and other respiratory diseases in full-term and premature infants (20).

There was an increased probability of PROM neonates needing to be treated in hospital. So, prolonging the period of hospitalization and increasing the in-hospital costs. The duration of hospitalization of neonates was prolonged by $20.0 \%$ in full-term infants and by $25.1 \%$ in premature infants with PROM, and the total in-hospital costs were increased by $30.5 \%$ in fullterm infants with PROM and by $60.0 \%$ in premature infants with PROM ${ }^{(21)}$.

\section{CONCLUSIONS}

We can conclude that, younger, illiterate parturient women were found to be provoking factors to increased PPROM. Such hazards may affect both maternal and neonatal outcomes such as infection, maternal distress, fetal distress, increased operative delivery as well as need for neonatal intensive care unit care in more than $50 \%$ of the neonates. Based on our results we recommend for further studies on larger patients and longer periods of follow up to emphasize our conclusion. Women diagnosed as having PROM should be hospitalized early and followed up until delivery with adequate neonatal intensive care unit.

\section{REFERENCES:}

1. Kiver V, Boos V, Thomas A et al. (2018): Perinatal outcomes after previable preterm premature rupture of membranes before 24 weeks of gestation. Journal of Perinatal Medicine, 46 (5): 555-565.

2. Maryuni M, Kurniasih D (2017): Risk factors of premature rupture of membrane. National Public Health Journal, 11 (3): 133-137.

3. Choudhary M, Rathore S, Chowdhary J et al. (2015): Pre and post conception risk factors in PROM. Int J Res Med Sci., 3 (10): 2594-2598.

4. Duncan J, Tobiasz A, Dorsett $K$ et al. (2020): Fetal pulmonary artery acceleration/ejection time prognostic accuracy for respiratory complications in preterm prelabor rupture of membranes. J Matern Fetal Neonatal Med., 33 (12): 2054-2058.

5. Negara K, Suwiyoga K, Arijana K et al. (2017): Role of Caspase-3 as risk factors of premature rupture of membranes. Biomedical and Pharmacology Journal, 10 (4): 2091-2098.

6. Negara S, Suwiyoga K, Arijana K et al. (2017): Role of Caspase-3 as Risk Factors of Premature Rupture of Membranes. Available from: http://biomedpharmajournal.org/?p=18124

7. Brian M, Mercer M (2003): Preterm Premature Rupture of the Membranes. The American College of Obstetricians and Gynecologists, 101: 1-6.

8. Okeke T, Enwereji J, Okoro O et al. (2014): The Incidencee and Management Outcome of Premature Rupture of Membranes (PPROM) in a Tertiary Hospital in Nigeria. American Journal of Clinical Medicine Research, 2 (1): 14-17.

9. Patil S, Patil V (2014): Maternal and foetal outcome in premature rupture of membranes. IOSR Journal of Dental and Medical Sciences, 13 (12): 56-83 
10. Noor S, Nazar A, Bashir R et al. (2007): Prevalence of PPROM and its outcome. J Ayub Med Coll Abbottabad, 19 (4): 14-7.

11. Vishwakarma K, Patel S, Yadav K et al., (2015): Impact of premature rupture of membranes on maternal $\&$ neonatal health in Central India. Journal of Evidence Based Medicine and Healthcare, 2 (49): 8505-8508.

12. Sae-Lin $P$, Wanitpongpan $P$ (2019): Incidence and risk factors of preterm premature rupture of membranes in singleton pregnancies at Siriraj Hospital. Journal of Obstetrics and Gynaecology Research, 45 (3): 573-577.

13. Bouvier D, Forest J, Blanchon L et al., (2019): Risk Factors and Outcomes of Preterm Premature Rupture of Membranes in a Cohort of 6968 Pregnant Women Prospectively Recruited. Journal of Clinical Medicine, 8 (11): 1987-93.

14. Whynott R, Vaught K, Segars J (2017): The Effect of Uterine Fibroids on Infertility: A Systematic Review. Semin Reprod Med., 35 (6): 523- 528.

15. Menon R, Richardson L (2017): Preterm prelabor rupture of the membranes: A disease of the fetal membranes. Semin Perinatol., 41 (7): 409-419.

16. Romero R, Miranda J, Chaemsaithong P et al. (2015): Sterile and microbial-associated intra-amniotic inflammation in preterm prelabor rupture of membranes J Matern Fetal Neonatal Med., 28 (12): 1394-409.

17. Andrews W, Goldenberg R, Hauth J et al. (2006): Interconceptional antibiotics to prevent spontaneous preterm birth: A randomized clinical trial. Am J Obstet Gynecol., 194: 617-623.

18. Adeniji A, Atanda $O$ (2013): Interventions and Neonatal Outcomes in Patients with Premature Rupture of Fetal Membranes at and Beyond 34 weeks Gestational Age at a Tertiary Health Facility in Nigeria. British Journal of Medicine \& Medical Research, 3 (4): 13881397.

19. Tchirikov M, Schlabritz-Loutsevitch N, Maher J et al. (2018): Mid-trimester preterm premature rupture of membranes (PPROM): etiology, diagnosis, classification, international recommendations of treatment options and outcome. J Perinat Med., 46 (5): 465-488.

20. Lorthe E (2018): Epidemiology, risk factors and child prognosis: CNGOF Preterm Premature Rupture of Membranes Guidelines. Gynecol Obstet Fertil Senol., 46 (12): 1004-1021.532.

21. Getahun D, Ananth C, Oyelese Y et al. (2007): Acute and chronic respiratory diseases in pregnancy: associations with spontaneous premature rupture of membranes. J Matern Fetal Neonatal Med., 20: 669-75.

22. Liu J, He J, Wang Q et al. (2005): The incidence of periventricular-intraventricular hemorrhage in preterm infants and its high-risk factors. Zhonghua Er Ke Za Zhi., 43: 216-17. 